\title{
Genotype-phenotype correlations in Peutz-Jeghers syndrome
}

\author{
C I Amos, M B Keitheri-Cheteri, M Sabripour, C Wei, T J McGarrity, M F Seldin, L Nations, \\ P M Lynch, H H Fidder, E Friedman, M L Frazier
}

J Med Genet 2004;41:327-333. doi: 10.1136/jmg.2003.010900

See end of article for authors' affiliations

.....................

Correspondence to: C Amos, PhD, Box 189,

Department of

Epidemiology, The

University of Texas

U.T. M.D. Anderson

Cancer Center, 1515

Holcombe Boulevard

Houston, TX 77030, USA;

camos@mdanderson.org

Revised version received 31 August 2003

Accepted for publication

5 September 2003 rome (PJS) is a dominantly inherited disorder often caused by Background and aims: Peutz-Jeghers syndrome (PJS) is a dominantly inherited disorder often caused by
mutations in STK1 1. Time to onset of symptoms was characterised for a large collection of individuals with PJS who had been tested for STK 11 mutations and genotype-phenotype correlations were evaluated. Methods: We characterised mutations in 42 independent probands and also used a historical cohort design to study 51 individuals with Peutz-Jeghers syndrome who had completed self-administered questionnaires.

Results: Mutations were detected in 22/32 (69\%) probands with PJS and 0/10 probands referred to rule out PJS. Real-time PCR analysis to quantitate DNA failed to detect any large deletions in PJS participants without STK 11 mutations. The median time to onset for gastrointestinal symptoms or polypectomy was 13 years of age but showed a wide variability. Gastric polyps were frequent in PJS participants, with a median age at onset of 16 years. Individuals with missense mutations had a significantly later time to onset of first polypectomy $(p=0.04)$ and of other symptoms compared with those participants either with truncating mutations or no detectable mutation.

Conclusion: STK 11 mutation analysis should be restricted to individuals who meet PJS criteria or their close relatives. Direct sequencing of STK 11 yields a high rate of point mutations in individuals who meet phenotypic PJS criteria. Individuals with missense mutations of STK 11 typically had a later time to onset for PJS symptoms. The common occurrence of gastric polyps may facilitate chemopreventive studies for this disorder.
$\mathrm{P}$ eutz-Jeghers syndrome (PJS) is a rare dominantly inherited disorder. The estimated frequency of the syndrome varies from $1 / 29000^{1}$ to $1 / 120000 .^{2}$ It is characterised by gastrointestinal (GI) hamartomatous polyps and mucocutaneous pigmentation. ${ }^{3}{ }^{4}$ A previous review found that $96 \%$ of patients develop hamartomatous polyps of the small bowel and polyps also commonly occur in the colon and stomach. ${ }^{5}$ Hamartomatous polyps can cause abdominal pain and self-limiting intussusception, sometimes causing bowel obstruction and severe gastrointestinal bleeding. ${ }^{6}$ In addition, buccal hypermelanocytic lesions were reported to occur in $93 \%$ of individuals with PJS. ${ }^{7}$ Because hyperpigmentation in PJS patients often fades with age, ${ }^{1}$ this sign is not necessarily reliably present for patients identified in adulthood.

Symptoms from polyps have a variable time to onset, which has not been well characterised. One study of 30 Korean patients found a median age at onset of first GI symptom of 12.5 years. ${ }^{7}$ Individuals with PJS have an increased risk for adenocarcinoma of the GI tract and extraintestinal malignancies such as breast, pancreas, and reproductive organs, particularly at early ages. ${ }^{8-11}$

The only known cause for PJS is mutation in STKll (alternatively known as LKBl) on chromosome 19p13.3..$^{12}$ However, linkage studies suggest that a minority of families with PJS do not have STKll mutations ${ }^{14}$ and loci on chromosomes $19 \mathrm{q}^{15}$ and $6 \mathrm{q}^{16}$ have been suggested in a few families. Specific genetic factors other than STK11 have not yet been identified. Although an STKll interacting protein (STKllIP) has been identified on chromosome 2, no mutations have yet been found in this or other candidate genes. ${ }^{17}$

Information is lacking about the natural history of PJS and its relationship to STKll mutations. We, therefore, implemented a historical cohort study to estimate time to onset of gastrointestinal symptoms such as abdominal pain, first GI bleeding, first intestinal intussusception, first detection of gastric polyps, first detection of non-colonic polyps, and polypectomies in PJS individuals. We also compared time to onset of PJS-affected individuals with and without detected STKll mutations.

\section{METHODS}

The study population included five probands with PeutzJeghers syndrome who were seen at the University of Texas M.D. Anderson Cancer Center, four probands from the M.S. Hershey Medical Center, Penn State College of Medicine, four probands referred by an onco-genetics service at the Sheba Medical Center in Israel, and 33 probands recruited through referrals to the University of Texas M.D. Anderson Cancer Center either from our website or from gastroenterologists and genetic professionals. Confirmed sporadic cases $(n=11)$ were defined as having no family history of Peutz-Jeghers syndrome, two or more hamartomatous polyps, and mucocutaneous hyperpigmentation. An additional three sporadic patients failed to meet the PJS criteria because we could not obtain documentation to show that they had hamartomatous polyps of the small bowel. These probands were labelled as likely to have PJS because they reported polyposis, had hyperpigmentation, and were referred by a medical professional. An additional three sporadic cases were labelled as possible PJS because they lacked documentation of two hamartomatous polyps and were self-referred or lacked hyperpigmentation. We identified seven confirmed familial cases that met the same criteria as the sporadic confirmed cases and also had a family history of Peutz-Jeghers

Abbreviations: $\mathrm{Gl}$, gastrointestinal; PJS, Peutz-Jeghers syndrome 
syndrome in a first degree relative. One familial case lacked hyperpigmentation but we classified this subject as a confirmed case based upon many documented PJS polyps.

An additional seven familial cases were labelled as likely because we did not obtain documentation from them. One suspected PJS case was included as a likely case because this subject had mucocutaneous hyperpigmentation and reported relatives with breast and colon cancer, including a sister with hamartomatous polyps and breast cancer. Blood samples were obtained from 32 PJS probands and 19 PJS affected relatives of these probands. Questionnaire data and sequence information were available from 10 individuals who were referred to rule out PJS. Included in this group were: two patients with breast cancer and a family history of cancer (one case was referred for p53 testing based upon a family history of very early onset breast cancer); one case with hyperplastic and hamartomatous polyps along with skin cysts who was referred for PTEN testing; five cases with a history of hyperpigmentation and GI symptoms but no history of polyposis (one case was deemed possible coeliac disease); one patient with a rectal hamartoma but no pigment; and one patient with a history of only hyperpigmentation. We were interested to identify whether some individuals with STK11 mutations might present with a milder spectrum of phenotypes insufficient for diagnosis of Peutz-Jeghers syndrome and so tested these individuals for STK11 mutations.

All participants were enrolled on an Institutional Review Board-approved protocol. Participants were asked to provide a detailed personal medical history questionnaire and blood after signing informed consent forms. We used a selfadministered questionnaire to collect information from both affected and unaffected family members, on which participants indicated the age at which they first had gastrointestinal symptoms, their first polypectomy, and other symptoms of PJS. At the same time we requested medical history information and access to pathological reports from all affected individuals. At least one family member was asked to fill out a detailed family history questionnaire. We recontacted participants who did not complete information about polypectomies. We obtained medical records from 30 of the 52 PJS cases.

\section{Mutation analysis}

For affected persons who gave blood, we directly sequenced STKll (LKBl) using the primers presented in table 1 to search for mutations in exons $1-3$ and 6-9, while exons 4 and 5 and the intervening intron were jointly sequenced. ${ }^{12}$ We modified the prior protocol of Jenne et al ${ }^{12}$ to split exon 1 into two segments. We did not sequence exon 10, which is in the 3' untranslated region. The PCR fragments were amplified using primer pairs specific for each of the nine exons, in a $30 \mu \mathrm{l}$ reaction mixture. The PCR products were mixed with $20 \mathrm{U}$ of exonuclease $\mathrm{l}$ and $4 \mathrm{U}$ of shrimp alkaline phosphatase and incubated at $37^{\circ} \mathrm{C}$ for $15 \mathrm{~min}$ and then at $85^{\circ} \mathrm{C}$ for $15 \mathrm{~min}$ to remove the unused primers and residual dNTPs. The products were subjected to electrophoresis in $1.5 \%$ agarose gels in TBE. Ethidium bromide was used to visualise the PCR products and to determine the fragment length and concentration. The DNA sequences of PCR products were sequenced in both directions using the forward and reverse primers. Sequencing was performed with an Applied Biosystems model 377 sequencer. We used the software application Polyphred (Phred, Phrap, and Consed) to detect polymorphisms and mutations. ${ }^{18-22} \mathrm{We}$ also visually checked all sequence runs to search for mutations that might have been missed by Polyphred analysis (none were found). When mutation detection identified a case in a family, all other relatives who showed features of PJS were assumed to have the same STKll mutation. Positive findings were verified in an independent CLIA certified laboratory, either at the U.T. M.D. Anderson Cancer Center or by GeneDx (Gaithersburg, MD, USA; http:// www.genedx.com/) so that the results could be disclosed to the proband's identified health care professional. There was complete concordance in mutation detection between the laboratories, except in one case in which a questionable missense mutation from analysis only in the forward sequencing direction (the reverse direction was negative) was definitively excluded by GeneDx.

For the 22 persons who had PJS, or were referred to rule out PJS, and who did not show detectable mutations from direct sequencing of STKll exons, we searched for larger deletions using real-time PCR. We designed the real-time PCR primers and probes for exon 1, exon 2, and exon 6 as shown in table 2. Blood DNA from a normal unaffected person was used as control. We used $12.5 \mathrm{ng}$ of template, $900 \mathrm{nM}$ of each primer, and $10 \mu \mathrm{M}$ probe. Multiplex PCR for both the STKll gene and RNase P gene as internal control were performed on an ABI Prism 7700 Sequence Detector (Applied Biosystems, Foster City, CA). A comparative $\left(\Delta \Delta \mathrm{C}_{\mathrm{T}}\right)$ method was used for relative quantitation of the gene copy number as suggested by the manufacturer. ${ }^{23}$

\section{Statistical analysis}

We used STATA software (STATA, College Station, TX, USA) for Kaplan-Meier analysis to characterise the time to onset of GI symptoms, polypectomies, gastric polyps, and intussusceptions, as shown in figs $1-4$. The log-rank test was used to test whether the time to onset of these symptoms varied by proband status (or was a relative in the pedigree), whether the case was familial or sporadic, and to compare participants with known STK11 mutations to those without detected mutation. We present analyses both without adjustment for possible correlation among the family members and after adjustment for family membership. For this adjustment, we used a sandwich estimator to correct for the common family membership of some participants ${ }^{24}$, in the presence of familial correlation with respect to time to onset. Data from individuals who failed to report an age at onset of any particular sign or symptom were deleted from the analysis of that condition. On figs $1-4$ we indicate the times at which individuals were censored (that is had not yet shown the condition of interest by their current age). The figures show the number of individuals censored at each age by including that figure above the time to onset line.

\section{RESULTS}

Summary characteristics of all participants who provided blood and completed questionnaires are shown in table 3. Of the probands, 15 had at least one other relative with PJS, while 17 were sporadic. We did not obtain histologic confirmation of two or more hamartomatous polyps from seven familial probands and six sporadic probands reported to have PJS. There were eight families in which either the proband or relatives had carcinomas. The relatively low proportion of cancers in the individuals we studied may reflect the relatively young ages of participants in our study. Of the familial probands $53 \%(8 / 15)$ were females while for the sporadic probands 53\% (9/17) were females. We obtained pathologicial confirmation from one of six referrals from genetic counsellors, 12 of 14 referrals from gastroenterologists, four of five referrals from geneticists, and two of seven self-referrals. Perhaps the lower rate of confirmation that we were able to achieve in referrals from genetic counsellors reflects the referral patterns in which genetic counsellors see patients from multiple sites. In some cases, we were not provided with sufficient information to retrieve documents. 


\begin{tabular}{|c|c|c|}
\hline Exon & Forward primer & Reverse primer \\
\hline 1 (1st half) & 5'-GGTCCCCGAGGACGAAGTGA & 5'-ATCAGGTACTTGCCGATGAG \\
\hline 1 (2nd half) & 5'-TCATCTACCAGCCGCGCCGCAA & 5'-ACCATCAGCACCGTGACTGG \\
\hline 2 & 5'-TCGCCGGCCGATGACAGA & 5'-AAGGAGACGGGAAGAGGAGCAG \\
\hline 3 & 5'-GAGGAGGGGCAAGGTGGGT & 5'-GTGTGGCCTCACGGAAAGGAC \\
\hline 4 & 5'-AGCTGGGCCTGTGGTGITG & 5'-CAGAGGCCCCTCGGAGTGTG \\
\hline 5 & 5'-AGCTGGGCCTGTGGTGTIT & 5'-CAGAGGCCCCTCGGAGTGTG \\
\hline 6 & 5'-TCAGTCCTCTCAATGCCTGCTG & 5'-GCCTCTGTCCCTGGGGTAGA \\
\hline 7 & 5'-GCGGGGTCCCCCTTAGGAG & 5'-CTAGCGCCCGCTCAACCAG \\
\hline 8 & 5'-GGAGCTGGGTCGGAAAACTGGA & 5'-TGCTCCCGTGGGACATCCTG \\
\hline 9 & 5'-GTAAGTGCGTCCCCGTGGTG & 5'-GTGGCATCCAGGCGITGTCC \\
\hline
\end{tabular}

We detected pathological mutations in $69 \%$ of independently tested probands (22/32). Of the detected mutations, six $(27 \%)$ were missense mutations, six $(27 \%)$ were insertions, four (18\%) were deletions, four were nonsense (18\%), one (5\%) affected a splice site, and one was likely to introduce alternate splicing. This proband had a silent mutation from GCG to GCA in codon 347 of exon 8 that may introduce a cryptic splice site. Interestingly, exon 8 uses the alternate (low-affinity) splicing acceptor sequence gca and it is possible that the cause of PJS in this individual (who developed an oesophageal carcinoma) might be aberrant splicing of this exon. Because the pathological significance of this variant is uncertain, we excluded data from this subject when comparing time to onset of symptoms of PJS according to whether participants were or were not carriers of known mutations in STK11.

For six patients we identified only polymorphic variants (in introns) and for four patients no mutations or polymorphisms were detected, so that in all $10 / 32(32 \%)$ did not have detectable mutations by sequence analysis. Of these, one is family 4 in our linkage study from which we previously found evidence for linkage to $19 \mathrm{p} 13.3 .{ }^{25}$ In addition to the participants from independent families, we also had questionnaire data from 19 PJS-affected participants in families, so that 51 participants in all had known mutation information and questionnaire data.

The inclusion criteria that we have used in this study could be deemed excessively liberal. Among those for whom we had confirmed PJS diagnoses, the proportion testing positive was $79 \%$. Mutations were detected in $73 \%(8 / 11)$ of patients who we scored as likely to have PJS. Notably, none $(0 / 3)$ of those that we have scored as possible PJS in table 3 tested positive for STK11 mutations. Possible status was assigned to these three patients because of a lack of confirmation of PJS polyps and a lack of hyperpigmentation (ID 41), self-referral (ID 38), and confirmation of only a single PJS polyp found after four polypectomies (ID 59). We failed to detect any mutations among the nine participants who were referred by genetic counsellors to rule out PJS based upon features

\begin{tabular}{ll}
$\begin{array}{l}\text { Table } 2 \\
\text { probes }\end{array}$ & Sequences for real-time PCR primers and \\
\hline Primer or probe & Sequence \\
\hline Exon 1 forward primer & TCGGTGGGTATGGACACGTT \\
Exon 1 reverse primer & GCGCGGCTGGTAGATGAC \\
Exon 1 probe & TCCACCGCATCGAC \\
Exon 2 forward primer & TGATACACCCTGTCCTCTCTG \\
Exon 2 reverse primer & TGTGCGTAACCTCCTCAGTAG \\
Exon 2 probe & CCAGGGAATTCA \\
Exon 6 forward primer & ACATCACCACGGGTCTACC \\
Exon 6 reverse primer & TGGCGTAGCTCCCTTCC \\
Exon 6 probe & ACATCTACAAGTTGTTGAGAAC \\
\hline
\end{tabular}

possibly consistent with PJS such as a family history of cancers or a single polyp.

\section{Protein modelling to evaluate missense mutations}

Protein models of STK11 were used to analyse the impact of missense mutations on STKll structure and function. Homology modelling was utilised to obtain a preliminary structure based on three kinase (1ATP, 1KOB, lAQ1) templates. ${ }^{15}$ Although these models rely on a predicted STKll structure, they can be of great benefit in understanding the role of STKll mutations in Peutz-Jeghers syndrome. Mutations R304W (ID 61) and L67P (ID 34) have been shown to disrupt auto-phosphorylation activity. ${ }^{13}{ }^{15}$ The F354L (ID 9) mutation has been reported, however, its impact on STKll activity is unknown. ${ }^{26}$ It is important to note that the F354L mutation occurs in the C-terminal region of the protein. This region contains a CAAX box, which is a consensus sequence for prenylation. ${ }^{27}$ Also, the C-terminal region contains serine and threonine residues that are phosphorylated. ${ }^{28}$ Thus, mutations in the C-terminal region might interrupt phosphorylation and post-translational events critical for STK1 l activity. In our study, this mutation was found in an extended family including many affected relatives and the change cosegregated perfectly with disease in our earlier linkage analysis. ${ }^{25}$

The Q170P (ID 1) mutation occurs near the end of an $\alpha$-helix in the kinase domain. Typically, the introduction of proline in an $\alpha$-helix induces a bend in the helix. The Q170P mutation is likely to induce a bend near the C-terminal end of the $\alpha$-helix. This bend may impact on substrate interactions and catalytic activity. This mutation was detected in a very large family that has been the basis of its own report ${ }^{29}$ and which shows cosegregation with PJS. A missense mutation next to codon 170, G171S, has been reported. ${ }^{26}$ The N181E mutation (ID 32) occurs near the active site of STK11. The proximity of the mutation to the active site and the replacement of a polar amino acid with a charged one could impact on the protein's kinase activity. A mutation in codon

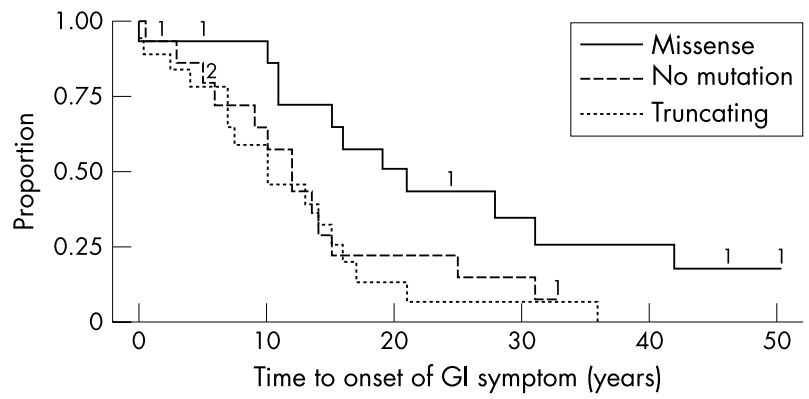

Figure 1 Time to onset of first reported gastrointestinal symptom by mutation group. 


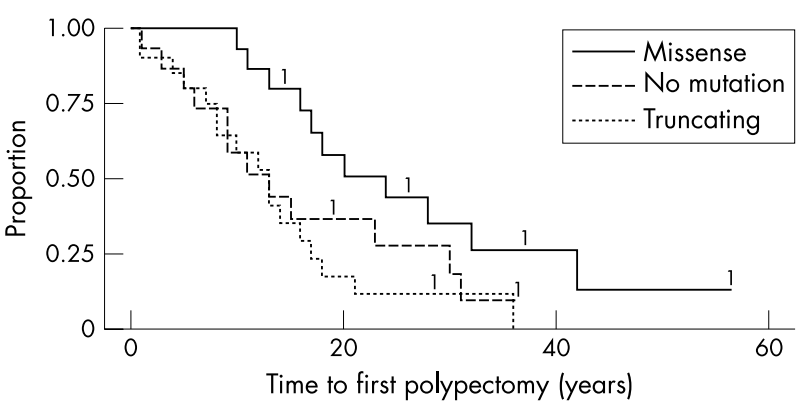

Figure 2 Time to onset of first polypectomy by mutation group.

181 has been reported (N181Y) but its impact is unknown. ${ }^{30}$ The T230P (ID 53) mutation occurs at a loop. Loops tend to be rich in charged and polar amino acids. The change to proline, a hydrophobic amino acid, likely induces a bend in the loop and impacts on substrate and solvent interactions. It is important to note that mutations near this region have been reported (S232P) but their impact is not clear. ${ }^{31}$

\section{Phenotypic characteristics}

About $82 \%(42 / 51)$ of those tested for STKll mutations reported hyperpigmentation. Among all individuals $(n=51)$ who had PJS with mutation results the median time to onset of GI symptoms and the median time to onset of first polypectomy were both 13 years of age (figs 1 and 2). There was a significant difference between time to onset of GI symptoms such as abdominal pain by mutation type as assessed by the log-rank test $(p=0.025)$, but further adjustment to allow for intrafamilial correlations reduced the significance of this finding $(p=0.07)$. The median times to onset of first reported GI symptoms were 21 years for individuals with missense mutations, 10 years of age for participants with truncating mutations, and 12 years of age for those PJS participants with undetectable mutations. Similarly, we found a borderline difference in time to onset of first polypectomy among the three mutation groups $(\mathrm{p}=0.05$ prior to family adjustment, $\mathrm{p}=0.08$ after adjustment). The median times to onset of first polypectomy were 28,18 , and 14 years of age respectively for participants with missense, truncating, or undetectable mutations. In addition, there was a significant difference between time to first detection of gastric polyps by mutation type as assessed by the log-rank test (fig 3; $\mathrm{p}=0.03, \mathrm{p}=0.09$ after adjustment). The median times were 23,13 , and 15 years for participants with missense, truncating, or undetectable mutations. Time to first intussusception (fig 4) also varied according to mutation status $(\mathrm{p}=0.04$ prior to adjustment, $\mathrm{p}=0.3$ after adjustment). Fewer than $50 \%$ of participants with missense mutations reported intussusceptions, while the median time to onset of this endpoint was 13 and 15 years of age for those

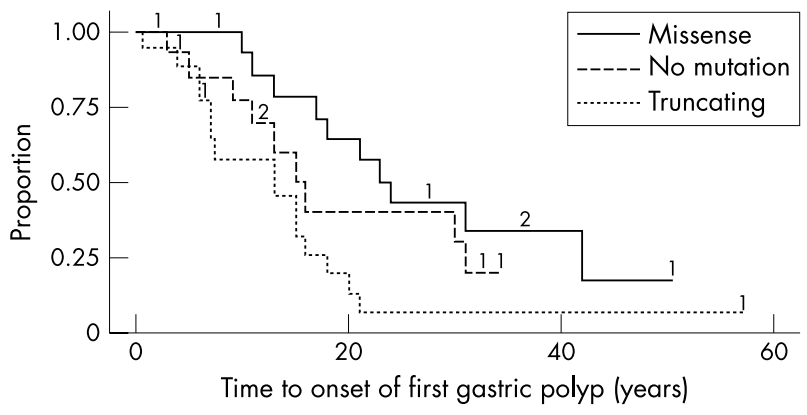

Figure 3 Time to onset of first gastric polyp by mutation group.

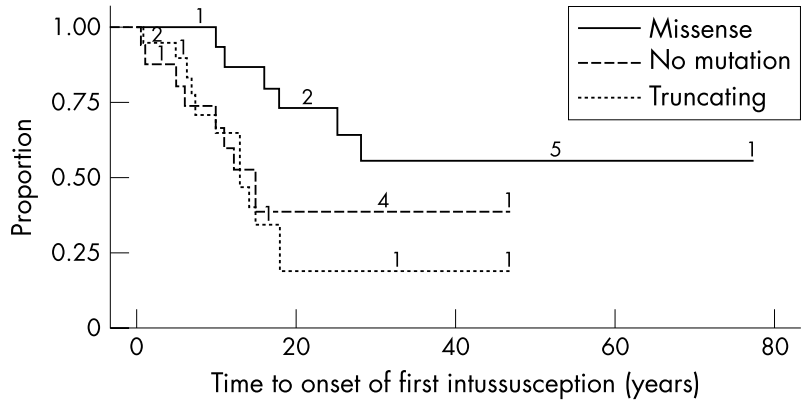

Figure 4 Time to onset of first intussusception by mutation group.

individuals with truncating or undetectable mutations. The large difference in significance prior to and after adjustment suggests strong familial effects in the endpoint of intussusception. Despite the borderline significance of some findings, the time to onset of the four symptoms described here varied considerably among groups for these conditions. We also evaluated the impact that including the three possible cases of PJS in our study might have had upon the time to onset analysis. When these three subjects were excluded, we still found significant differences among the mutation groups for time to polypectomy $(\mathrm{p}=0.03)$, first intussusception $(\mathrm{p}=0.04)$, and time to gastric polyps $(\mathrm{p}=0.06)$.

To assess the possibility that individuals with the most severe phenotype had been referred as probands into our study, we compared the time to onset of probands versus relatives with detected mutations. For these two groups we found a similar time to onset of the major signs and symptoms we studied, including time to onset of first gastrointestinal symptoms, age at first polypectomy, and time to onset of gastric polyps. This finding indicates that the selection of probands into our study was not strongly correlated with these symptoms. This lack of correlation suggests that our findings are generalisable to other populations of Peutz-Jeghers patients, since the selection process appears uncorrelated with the disease endpoints we are studying. We similarly did not find significant differences according to whether the proband was familial or sporadic with respect to the time to onset of the four symptoms evaluated.

\section{DISCUSSION}

The success of mutation detection varied according to the reason for referral. As we did not detect any STK11 mutations among nine individuals referred to rule out PJS in this study, we do not believe that STKIl mutation testing is warranted for participants who fail to meet the rather broad definition of PJS we used in our study. On the other hand, we did identify STK11 mutations in most study participants with PJS, suggesting that STK11 mutation analysis is effective in this group. Furthermore, despite the small sample size, we have identified significant differences in the time to onset of symptoms of PJS associated with the type of STKll mutation that we identified. A priori, we might have expected that participants who did not show STKll mutations might have a less severe manifestation of PJS. We anticipate that some individuals with PJS harbour currently undetectable mutations in the regulatory regions of STKll. At least some apparent regulatory variants, such as that inferred in family 4 with ID 6 in this study ${ }^{25}$, result in a phenotype similar to that observed among participants with truncating mutations of STK11. Alternately, mutations in genes other that STK11 may cause a similarly severe form of PJS as found for individuals with truncating mutations. A recent report indicates an association of hyperpigmentation and gynaecological 


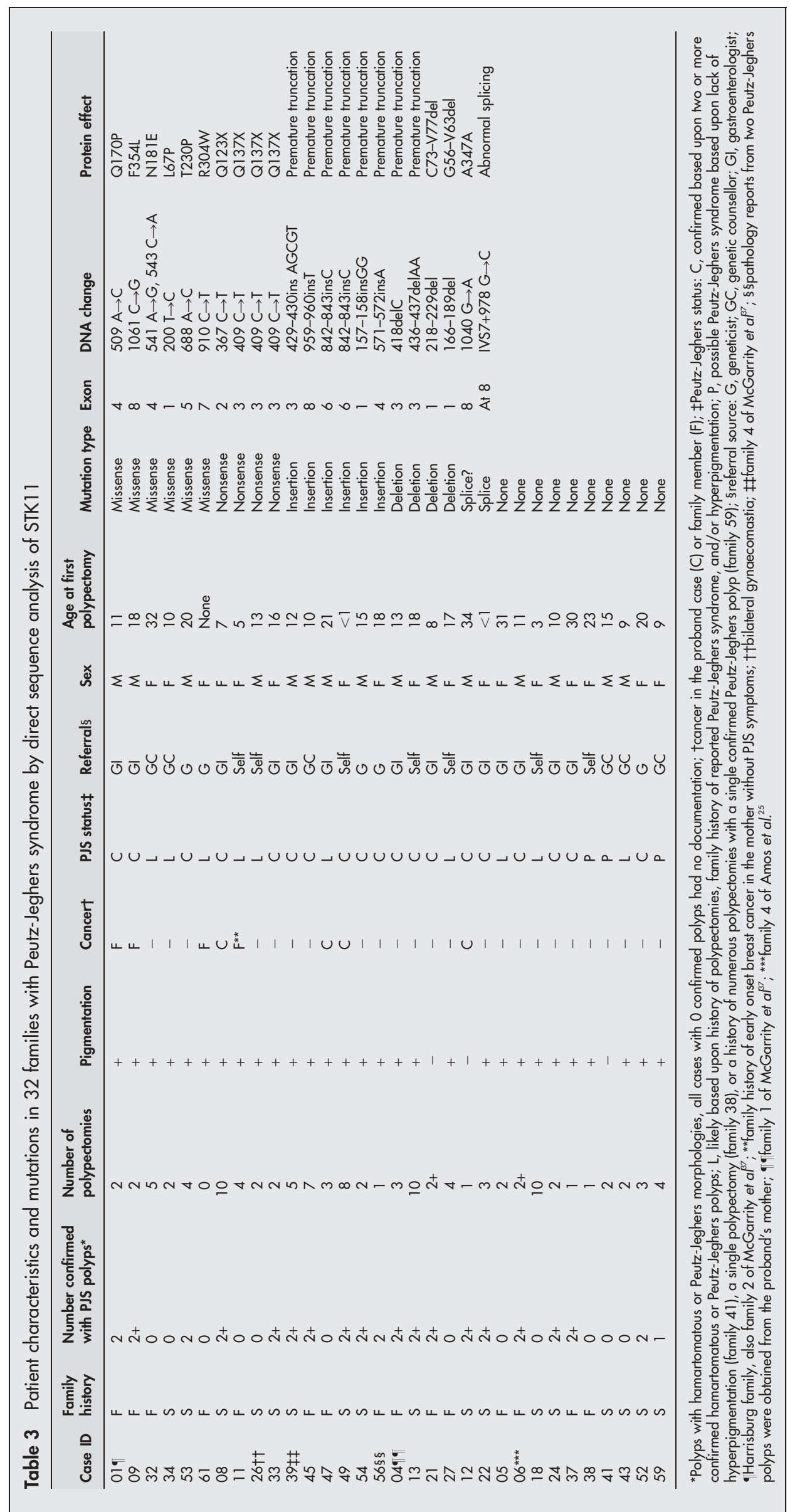


cancers. $^{32}$ These observations suggest that if another locus predisposes for PJS or PJS-like conditions, it may have a more pronounced impact in women. Several other reports have indicated other loci than STKll may cause PJS. ${ }^{14} 15173334$ Our study suggests that these other genetic causes explain a minority of cases of PJS.

Our study obtained participants from a variety of sources and reflects referral to tertiary care centres. In order to evaluate potential selection bias we compared the time to onset among probands with their relatives, and did not find any significant differences. We also did not find any significant difference in time to onset between familial probands and sporadic probands. These results argue against selection bias strongly affecting our results. If selection bias were a major determinant of time to onset, then we would have anticipated that familial probands would have an earlier time to onset than their relatives. Mutation status was unknown prior to the enrolment of participants into our study. Therefore, any selection of participants into the study would not explain the differences we observed in time to onset according to the types of mutations participants had. The selection of cases through individuals affected with symptoms may still bias the sample to contain mutations having severe phenotypic effects. In most of the PJS families evaluated, differences existed between exon sites and types of mutation. This is the first study to examine the time to onset of GI symptoms and polypectomies in a large sample that had been tested for STK1l mutations. Our results show early onset of polypectomies, first gastrointestinal symptoms, and gastric polyps. Our finding that gastric polyps are very common among individuals with PJS has not been properly appreciated in the genetics community and provides an opportunity for further chemopreventive studies.

The mechanisms involved in the tumour suppressor function of STKll in PJS remain largely uncharacterised. However, cyclooxygenase $2(\mathrm{COX}-2)$ plays a key role in inflammatory response and cell cycle control. Chemoprevention studies have shown that down regulating COX-2 in participants with familial adenomatous polyposis controls adenomatous polyp growth. ${ }^{35}$ Similarly, clinical chemoprevention studies have shown a strong protective role of aspirin, which down regulates COX-2, in protecting from colon cancer. ${ }^{36}$ Our recent studies showed that COX-2 levels were increased in a majority of hamartomatous polyps from patients with PJS ${ }^{37}{ }^{38}$ and similar results have been found in animal models. ${ }^{39}$ These findings suggest that COX-2 modulation might be important for either controlling hamartomatous growth or decreasing cancer risk among participants with PJS. Our study shows that the majority of participants with PJS show gastric polyps. The ease in accessing this site for visualisation and measurement in chemopreventive studies indicates the feasibility of clinical trials of COX-2 and other potential chemopreventive agents.

The variability of time to onset of symptoms according to the type of STKll mutation (missense versus truncating) suggests a potential value for genetic testing of STKll in disease management. However, larger studies would help to evaluate the strength of correlation in time to onset within families, and to identify whether any missense mutations have particularly later or earlier times to onset of symptoms.

\section{ACKNOWLEDGEMENTS}

We thank those who participated in our research and Stephanie Sugars.

\footnotetext{
Authors' affiliations

C I Amos, M B Keitheri-Cheteri, M Sabripour, C Wei, L Nations,

M L Frazier, Department of Epidemiology, U.T. M.D. Anderson Cancer Center HMB, Houston, TX, USA
}

T J McGarrity, Division of Gastroenterology and Hepatology, Penn State College of Medicine, Milton S. Hershey Medical Center, Hershey, PA, USA

M F Seldin, Rowe Program in Genetics, UC Davis, CA, USA

P M Lynch, Department of GI Medicine and Nutrition, U.T. M.D.

Anderson Cancer Center HMB, Houston, TX, USA

H H Fidder, Institute of Gastroenterology, Sheba Medical Center, Tel-

Hashomer, 52621, Israel

E Friedman, The Susanne Levy Gerner Oncogenetics Unit, Sheba Medical Center, Tel-Hashomer, 52621, Israel

This research has been partially supported by grants ACS/RPG99-030$01-\mathrm{CCE}-3$ and $\mathrm{NIH} / \mathrm{NCl} \mathrm{CA} 78142$.

Conflicts of interest: none declared.

\section{REFERENCES}

1 Finan MC, Ray MK. Gastrointestinal polyposis syndromes. Dermatol Clin 1989:7:419-34

2 Linder NM, Greene MH. The concise handbook of family cancer syndromes. J Natl Cancer Inst 1998;90:1039-71.

3 Jeghers H, McKusick VA, Katz KH. Generalized intestinal polyposis and melanin spots of the oral mucosa, lips and digits. N Engl J Med 1949;241:993-1005.

4 McGarrity TJ, Kulin HK, Zaino RJ. Peutz-Jeghers syndrome. Am J Gastroenterol 2000;95:596-604.

5 Bartholomew LG, Dahlin DC, Waugh JM. Intestinal polyposis associated with mucocutaneous melanin pigmentation (Peutz-Jeghers syndrome). Review of the literature and report of six cases with special reference to pathologic findings. Gastroenterology 1956;32:434-51.

6 Spigelman AD, Arese P, Phillips RKS. Polyposis: the Peutz-Jeghers syndrome. Br J Surg 1995:82:1311-4.

7 Choi HS, Park YG, Youk EG, Yoon K-A, Ku J-L, Kim NK, Kim SM, Kim YJ, Moon DJ, Min JS, Park CJ, Bae OS, Yang D-H, Jun SH, Chung ES, Jung PM, Whang Y, Park J-G. Clinical characteristics of Peutz-Jeghers syndrome in Korean polyposis patients. Int J Colorectal Dis 2000;15:35-8.

8 Giardiello FM, Welsh SB, Offerhaus GJA, Booker SV, Krush AJ, Hamilton SR, Yardley JH, Luk GD. Increased risk of cancer in Peutz-Jeghers syndrome. N Engl J Med 1987;316:151-4.

9 Hizawa K, lida M, Matsumoto T, Kohrogi N, Kinoshita H, Yao T, Fujishima M. Cancer in Peutz-Jeghers syndrome. Cancer 1993;72:2777-81.

10 Boardman LA, Thibodeau SN, Schaid DJ, Lindor NM, McDonnell SK, Burgart $\sqcup$, Ahlquist DA, Podratz KC, Pittelkow M, Hartmann LC. Increased risk for cancer in patients with the Peutz-Jeghers syndrome. Ann Intern Med 1998; 128:896-9.

11 Giardiello FM, Brensinger JD, Tersmette AC, Goodman SN, Petersen GM, Booker SV, Cruz-Correa M, Offerhaus JA. Very high risk of cancer in familial Peutz-Jeghers syndrome. Gastroenterology 2000;1 19:1447-53.

12 Jenne DE, Reimann H, Nezu J, Friedel W, Loff S, Jeschke R, Muller O, Back W, Zimmer M. Peutz-Jeghers syndrome is caused by mutations in a novel serine threonine kinase. Nat Genet 1998;18:38-43.

13 Hemminki A, Markie D, Tomlinson I, Avizienyte E, Roth S, Loukola A, Bignell G, Warren W, Aminoff M, Hoglund P, Jarvinen H, Kristo P, Pelin K, Ridanpaa M, Salovaara R, Toro T, Bodmer W, Olschwang S, Olsen AS, Stratton MR, de la Chapelle A, Aaltonen LA. A serine/threonine kinase gene defective in Peutz-Jeghers syndrome. Nature 1998;391:184-7.

14 Olschwang S, Markie D, Seal S, Neale K, Phillips R, Cottrell S, Ellis I, Hodgson S, Zauber P, Spigelman A, Iwama T, Loff S, McKeown C, Marchese C, Sampson J, Davies S, Talbot I, Wyke J, Thomas G, Bodmer W, Hemminki A, Avizienyte E, de la Chapelle A, Aaltonen L, Tomlinson I. PeutzJeghers disease: most, but not all, families are compatible with linkage to 19p13.3. J Med Genet 1998;35:42-4

15 Mehenni H, Gehrig C, Nezu J, Oku A, Shimane M, Rossier C, Guex N, Blouin JL, Scott HS, Antonarakis SE. Loss of LKB1 kinase activity in PeutzJeghers syndrome, and evidence for allelic and locus heterogeneity. Am J Hum Genet 1998:63:1641-50.

16 Tomlinson IP, Olschwang S, Abelovitch D, Nakamura Y, Bodmer WF, Thomas G, Markie D. Testing candidate loci on chromosomes 1 and 6 for genetic linkage to Peutz-Jeghers' disease. Ann Hum Genet 1996:60:377-84.

17 Buchet-Poyau K, Mehenni H, Radhakrishna U, Antonarakis SE. Search for the second Peutz-Jeghers syndrome locus: exclusion of the STK13, PRKCG, KLK10, and PSCD2 genes on chromosome 19 and the STK1 IIP gene on chromosome 2. Cytogenet Genome Res 2002;97:171-8.

18 Nickerson DA, Tobe VO, Taylor SL. Polyphred: automating the detection and genotyping of single nucleotide substitutions using fluorescence-based resequencing. Nucleic Acids Res 1997;25:2745-51.

19 Ewing B, Hillier L, Wendl M, Green P. Basecalling of automated sequencer traces using phred. I. Accuracy assessment. Genome Res 1998:8:175-85.

20 Ewing B, Green P. Basecalling of automated sequencer traces using phred. II. Error probabilities. Genome Res 1998;8:186-94.

21 Green P. Phrap, unpublished. http://www.genome.washington.edu.

22 Gordon D, Abajian C, Green P. Consed: a graphical tool for sequence finishing. Genome Res 1998;8:195-202.

23 ABI Prism, User Bulletin \#2: ABI Prism 7700 Sequence Detection System. Foster City, CA: ABI Prism, 2001:11-24.

24 Lipsitz SR, Parzen M. A jackknife estimator of variance for Cox regression for correlated survival data. Biometrics 1996;52:291-8. 
25 Amos CL, Bali D, Thiel TJ, Anderson JP, Gourley I, Frazier ML, Lynch PM, Luchtefeld MA, Young A, McGarrity TJ, Seldin MF. Fine mapping of a genetic locus for Peutz-Jeghers syndrome on chromosome 19p. Cancer Res 1997:57:3653-6

26 Dong SM, Kim KM, Kim SY, Shin MS, Na EY, Lee SH, Park WS, Yoo NJ, Jang JJ, Yoon CY, Kim JW, Kim SY, Yang YM, Kim SH, Kim CS, Lee JY. Frequent somatic mutations in serine/threonine kinase $11 /$ Peutz-Jeghers syndrome gene in left-sided colon cancer. Cancer Res 1998;58:3787-90.

27 Yoo LI, Chung DC, Yuan J. LKB1 - a master tumour suppressor of the small intestine and beyond. Nat Rev Cancer 2002;2:529-35.

28 Boudeau J, Sapkota G, Alessi DR. LKB1 a protein kinase regulating cell proliferation and polarity. FEBS Lett 2003;546:159-65.

29 Foley TR, McGarrity TJ, Abt AB. Peutz-Jeghers syndrome: a clinicopathologic survey of the "Harrisburg family" with a 49-year follow-up. Gastroenterology 1988:95: 1535-40

30 Ylikorkala A, Avizienyte E, Tomlinson IP, Tiainen M, Roth S, Loukola A, Hemminki A, Johansson M, Sistonen P, Markie D, Neale K, Phillips R, Zauber P, Twama T, Sampson J, Jarvinen H, Makela TP, Aaltonen LA Mutations and impaired function of LKBI in familial and non-familial PeutzJeghers syndrome and a sporadic testicular cancer. Hum Mol Genet 1999;8:45-51

31 Yoon KA, Ku JL, Choi HS, Heo SC, Jeong SY, Park YJ, Kim NK, Kim JC, Jung PM, Park JG. Germline mutations of the STK11 gene in Korean PeutzJeghers syndrome patients. Br J Cancer 2000;82:1403-6.

32 Boardman LA, Pittelkow MR, Couch FJ, Schaid DJ, McDonnell SK, Burgart U, Ahlquist DA, Carney JA, Schwartz DI, Thibodeau SN, Hartmann LC.
Association of Peutz-Jeghers-like mucocutaneous pigmentation with breast and gynecologic carcinomas in women. Medicine 2000;79:293-8.

33 Boardman LA, Couch FJ, Burgart L, et al. Genetic heterogeneity in PeutzJeghers syndrome. Hum Mut 2000;16:23-30.

34 Olschwang S, Boisson C, Thomas G. Peutz-Jeghers families unlinked to STK 1 1/LKB 1 gene mutations are highly predisposed to primitive biliary adenocarcinoma. J Med Genet 2001;38:356-60.

35 Steinbach G, Lynch PM, Phillips RK, Wallace MH, Hawk E, Gordon GB Wakabayashi N, Saunders B, Shen Y, Fujimura T, Su LK, Levin B. The effect of celecoxib, a cyclooxygenase- 2 inhibitor, in familial adenomatous polyposis. N Engl J Med 2000;342:1946-52.

36 Sandler RS, Halabi S, Baron JA, Budinger S, Paskett E, Keresztes R, Petrelli N, Pipas JM, Karp DD, Loprinzi CL, Steinbach G, Schilsky R. A randomized tria of aspirin to prevent colorectal adenomas in patients with previous colorectal cancer. N Engl J Med 2003;348:883-90.

37 McGarrity TJ, Peiffer L, Amos Cl, Frazier ML, Ward MG, Howett MK. Overexpression of cyclooxygenase 2 in hamartomatous polyps in PeutzJeghers syndrome. Gastroenterology 2003;98:671-8.

38 Wei C Amos Cl, Rashid A, Sabripour M, Nations L, McGarrity TJ, Frazier ML. Correlation of staining for LKB1 and COX-2 in hamartomatous polyps and carcinomas from patients with Peutz-Jeghers syndrome. J Histochem Cytochem 2003;51:1665-72.

39 Rossi DJ, Ylikorkala A, Korsisaari N, Salovaara R, Luukko K, Launonen V Henkemeyer M, Risimäki A, Aaltonen LA, Mäkel TP. Induction of cyclooxygenase-2 in a mouse model of Peutz-Jeghers polyposis. Proc Natl Acad Sci USA 2002;99:12327-32. 